Service social

\title{
L'implantation d'un système informatisé des dossiers de service social en milieu hospitalier
}

\section{Georgette Béliveau, Martin Poulin et Ginette Beaudoin}

Volume 41, numéro 1, 1992

L'avenir des services ou services d'avenir

URI : https://id.erudit.org/iderudit/706560ar

DOI : https://doi.org/10.7202/706560ar

Aller au sommaire du numéro

Éditeur(s)

École de service social de l'Université Laval

ISSN

1708-1734 (numérique)

Découvrir la revue

Citer cet article

Béliveau, G., Poulin, M. \& Beaudoin, G. (1992). L'implantation d'un système informatisé des dossiers de service social en milieu hospitalier. Service social, 41(1), 104-126. https://doi.org/10.7202/706560ar
Résumé de l'article

Cet article rapporte les résultats d'une recherche portant sur le processus d'implantation de l'informatisation des données de service social en milieu hospitalier. L'implantation de l'informatique a été étudiée dans quatre cliniques pédiatriques d'un centre hospitalier. Il s'agit d'une étude longitudinale, d'une durée de trois ans et qui se classe dans la catégorie des études descriptives à caractère exploratoire. Cette étude de cas, de nature qualitative, vise principalement à observer le phénomène d'implantation tel qu'il se déroule. Les auteurs présentent d'abord leur modèle théorique des phases d'implantation, puis la collecte et l'analyse des données. Enfin, la conclusion fait ressortir ce qu'il semble pertinent de retenir de cette expérience. 
Georgette Béliveau, professeure titulaire, École de service social, Université Laval.

Martin Poulin, professeur titulaire, École de service social, Université Laval.

Ginette Beaudoin, professionnelle de recherche, Université Laval.

\section{L'implantation d'un système informatisé des dossiers de service social en milieu hospitalier ${ }^{1}$}

Georgette Béliveau Martin Poulin

Ginette Beaudoin

Notre première recherche sur l'utilisation de l'informatique en service social menée en 1987 abordait la question sous l'angle des perceptions et des attitudes des intervenants sociaux. Les résultats nous ont permis d'observer notamment que les praticiens sociaux, malgré l'expression de certaines résistances, possèdent généralement une attitude réceptive envers les applications de l'informatique dans la pratique professionnelle. A cet égard, le processus d'informatisation des données de service social en cours à l'hôpital Sainte-Justine de Montréal constitue un bon exemple d'une nouvelle application technologique à la pratique du service social. En effet, deux intervenantes sociales rattachées à cet établissement, Françoise Turgeon-Krawczuk et Claire Lalande-Gendreau, ont conçu un logiciel d'intervention familiale et sociale visant à informatiser les données de service social et à les intégrer aux données médicales. Cette initiative d'avant-garde se situe dans le courant du développement de l'usage de l'informatique dans les services sociaux. On se rappellera que dans ce domaine l'informatique a été d'abord utilisée pour la gestion. Depuis quelques années, on voit poindre certaines applications à la pratique professionnelle. Pour une vue d'ensemble de l'évolution, le lecteur peut 
se reporter aux ouvrages antérieurs des auteurs : Béliveau, Poulin et Beaudoin (1991 et 1987), Poulin, Beaudoin, Béliveau et al. (1991), Poulin et Béliveau (1987).

L'originalité de la démarche et les effets engendrés par une telle entreprise ne pouvaient que susciter notre intérêt en tant que professionnels et chercheurs en service social. Nous avons donc fait nôtre le souhait des intervenantes sociales d'accompagner ce projet d'implantation d'une recherche afin d'acquérir une meilleure connaissance des étapes inhérentes au processus d'implantation de l'informatique et de préciser la nature des répercussions dans le milieu de travail.

La partie de la recherche que nous rapportons dans cet article s'intéresse plus particulièrement au processus d'implantation de l'informatisation des données de service social en milieu hospitalier. Dans une publication ultérieure, nous avons I'intention de présenter les résultats concernant les effets de cette expérience. L'implantation de l'informatique a été étudiée dans quatre cliniques pédiatriques du centre hospitalier.

Il s'agit d'une étude longitudinale qui s'est échelonnée sur trois ans (juin 1988 à juin 1991). Elle se classe dans la catégorie des études descriptives à caractère exploratoire. Cette étude de cas, de nature qualitative, vise principalement à observer le phénomène d'implantation tel qu'il se déroule. Elle ne suppose pas l'élaboration d'un cadre théorique traditionnel ni d'hypothèses de départ. Pour ce faire, nous avons suivi la méthode de travail que Yin (1984) a élaborée au sujet des recherches qualitatives qui, dit-il, même si elles paraissent simples, ne sont jamais faciles à réaliser. La première démarche que cet auteur suggère est de préparer une grille de collecte de données qui s'inspire d'auteurs connus tout en l'adaptant à notre sujet. C'est ce que nous présentons au paragraphe intitulé "Les phases d'implantation de l'informatique ». Les pages qui portent sur la " collecte et l'analyse des données" expliquent succinctement la méthode de collecte des informations et la façon de les analyser, alors que la section suivante expose les différentes phases d'analyse des observations recueillies. Enfin, nous concluons par ce que nous croyons pertinent de retenir de cette expérience.

\section{Les phases d'implantation de l'informatique}

L'implantation de l'informatique dans les organisations ne s'opère pas selon un modèle théorique unique. La littérature est riche en modèles d'élaboration des phases ou des étapes à suivre. Les auteurs se penchent sur les étapes à franchir dans un proces- 
sus d'informatisation ou proposent des recommandations en vue d'assurer le succès de l'implantation de l'informatique, mais peu définissent le processus d'implantation. Il est clair toutefois que l'implantation de l'informatique devrait suivre un processus en plusieurs phases ou étapes. Ce qui nous amène à la définition suivante proposée par Larsen (1987) : un processus d'implantation consiste en une série d'étapes, chacune caractérisée par une chaîne d'actions et d'événements.

Dans cette étude, nous décrivons le processus d'implantation du système informatisé en nous basant sur les phases d'implantation décrites par Larsen (1987). Ces phases, habituellement expérimentées par les organisations de santé mentale, sont au nombre de quatre : la phase préparatoire, la phase d'implantation, la phase de clarification, la phase de routine.

La phase préparatoire associe le problème relevé avec la solution technologique proposée. L'organisation examine le matériel et les logiciels afin de déterminer quel système informatique peut répondre aux besoins de l'organisation. Les activités associées à cette phase consistent à recueillir de l'information sur les systèmes et les logiciels existants, à considérer la contribution d'un système aux besoins de l'organisation, à choisir la solution technologique appropriée, à planifier une éventuelle introduction de l'informatique et à fournir une assistance aux usagers possibles.

Les activités de la phase préparatoire constituent en quelque sorte un préalable à l'implantation de l'informatique. Les activités de la phase d'implantation se résument à : l'achat, la réception et l'installation du matériel informatique; l'information active et l'assistance technique aux usagers; la modification du système pour répondre aux besoins de l'organisation et l'ajustement de l'organisation.

Graduellement, plusieurs employés commencent à utiliser l'ordinateur et les capacités du système deviennent apparentes dans l'organisation. C'est le début de la phase de clarification. À cette étape, se précisent de façon plus permanente les modes

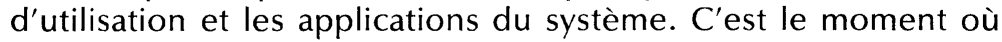
l'innovation apparait dans l'organisation et y prend graduellement sa place. À la phase de routine, l'ordinateur a été incorporé aux activités de l'organisation et n'est plus considéré comme une innovation.

Selon Larsen, ces étapes ne se suivent pas toujours dans une séquence aussi ordonnée. De même, une étape n'est pas toujours terminée avant que la prochaine ne débute. Les différents éléments de chacune des phases se chevauchent durant tout le processus d'implantation. Pour préciser les activités rattachées à chacune 
des phases, nous nous sommes référés au modèle de Larsen et aux autres auteurs qui ont traité le sujet (Cotter, 1981; Schoech, Schkade et Mayers, 1981; Hurtubise, 1984; Moulin, 1986). Le processus d'implantation a été étudié à partir du modèle présenté au tableau 1.

Selon le modèle que nous avons construit, les activités de la phase préparatoire numérotées de 1 à 6 inclusivement devraient se dérouler "idéalement "selon cet ordre. Par ailleurs, les quatre dernières activités, désignées comme étant $A, B, C$ et $D$, devant débuter également à la phase préparatoire, pourraient se dérouler dans un ordre différent de celui présenté dans le tableau 1 et s'intercaler entre les activités 1 à 6 . Cela n'empêche pas, comme Larsen (1983) le note, que les différents éléments de la phase

\section{TABLEAU 1}

\section{Processus d'implantation : phases et activités}

\begin{tabular}{|c|c|}
\hline Phases & Activités \\
\hline 1) Phase préparatoire & $\begin{array}{l}\text { 1. Mise en route du projet } \\
\text { 2. Définition des besoins } \\
\text { 3. Détermination des buts et objectifs } \\
\text { 4. Collecte d'informations } \\
\text { 5. Conception du système } \\
\text { 6. Planification de l'introduction du système } \\
\text { A) Préparation d'un plan de développement } \\
\text { B) Engagement de spécialistes en informatique } \\
\text { C) Définition de politiques de confidentialité et } \\
\text { de mécanismes de sécurité } \\
\text { D) Planification du budget }\end{array}$ \\
\hline II) Phase d'implantation & $\begin{array}{l}\text { 1. Mise en place du système : } \\
\text { - installation } \\
\text { - formation et assistance du personnel } \\
\text { 2. Mise en fonction et suivi du système : } \\
\text { - utilisation du système } \\
\text { - modification du système } \\
\text { - ajustements de l'organisation }\end{array}$ \\
\hline III) Phase de clarification & $\begin{array}{l}\text { Détermination d'arrangements plus staoles pour l'utili- } \\
\text { sation et les applications du système }\end{array}$ \\
\hline IV) Phase de routine & $\begin{array}{l}\text { Incorporation du système dans les activités de l'orga- } \\
\text { nisation. }\end{array}$ \\
\hline
\end{tabular}


préparatoire puissent se "poursuivre " à d'autres moments et qu'une étape ne soit pas toujours terminée avant que la suivante soit amorcée.

La description des activités est basée sur les écrits de : Cotter (1981), Hurtubise (1984), Larsen (1987), Duribreux (1988). Dans ce qui suit, nous définissons brièvement les différentes activités propres aux quatre phases et précisons leur mode d'opérationalisation. Les dix activités de la phase préparatoire (phase l) sont présentées dans le tableau 2, alors que les activités des autres phases sont décrites dans les paragraphes qui suivent ce tableau.

\section{TABLEAU 2}

\section{Définition et opérationalisation des activités de la phase I}

\begin{tabular}{|l|l|l|}
\hline \multicolumn{1}{|c|}{ Activités } & \multicolumn{1}{|c|}{ Définitions } & \multicolumn{1}{|c|}{$\begin{array}{c}\text { Indicateurs servant à } \\
\text { l'opérationalisation }\end{array}$} \\
\hline 1. Mise en route & $\begin{array}{l}\text { Différents moyens mis en } \\
\text { ceuvre dans le but de } \\
\text { démarrer le projet }\end{array}$ & $\begin{array}{l}\text { Description des différents } \\
\text { moyens employés pour } \\
\text { démarrer le projet et des } \\
\text { personnes qui sont à son } \\
\text { origine }\end{array}$ \\
\hline 2. Définition des besoins & $\begin{array}{l}\text { Analyse où sont précisés } \\
\text { les besoins, les données à } \\
\text { informatiser, les solutions } \\
\text { possibles }\end{array}$ & $\begin{array}{l}\text { Description de la nature } \\
\text { des besoins, des person- } \\
\text { nes engagées dans cette } \\
\text { tâche et des documents } \\
\text { où les besoins sont définis }\end{array}$ \\
\hline $\begin{array}{l}\text { 3. Détermination des buts } \\
\text { et des objectifs }\end{array}$ & $\begin{array}{l}\text { Ce que l'on envisage de } \\
\text { faire ou d'améliorer grâce } \\
\text { à l'informatisation }\end{array}$ & $\begin{array}{l}\text { Description de la nature } \\
\text { des buts et des objectifs } \\
\text { visés }\end{array}$ \\
\hline 4. Collecte d'informations & $\begin{array}{l}\text { Collecte d'informations } \\
\text { sur les systèmes et les } \\
\text { logiciels existants }\end{array}$ & $\begin{array}{l}\text { Description des démar- } \\
\text { ches de collecte : consul- } \\
\text { tation de documents, de } \\
\text { personnes-ressources et } \\
\text { analyses }\end{array}$ \\
\hline 5. Conception du système & $\begin{array}{l}\text { Conception et dévelop- } \\
\text { pement du système : } \\
\text { développement des } \\
\text { logiciels et des procédures } \\
\text { d'utilisation }\end{array}$ & $\begin{array}{l}\text { Description des personnes } \\
\text { engagées dans cette } \\
\text { tâche, du temps d'élabora- } \\
\text { tion et du développement } \\
\text { des étapes }\end{array}$ \\
\hline
\end{tabular}




\begin{tabular}{|c|l|l|}
\hline \multicolumn{1}{|c|}{ Activités } & \multicolumn{1}{|c|}{ Définitions } & \multicolumn{1}{|c|}{$\begin{array}{c}\text { Indicateurs servant à } \\
\text { l'opérationalisation }\end{array}$} \\
\hline $\begin{array}{l}\text { 6. Planification de l'in- } \\
\text { troduction du système }\end{array}$ & $\begin{array}{l}\text { Préparatifs et démarches } \\
\text { de planification visant à } \\
\text { installer le matériel à } \\
\text { venir, à informer et for- } \\
\text { mer les usagers potentiels }\end{array}$ & $\begin{array}{l}\text { Description des moyens } \\
\text { employés pour introduire } \\
\text { le système }\end{array}$ \\
\hline $\begin{array}{l}\text { A. Préparation d'un plan } \\
\text { de développement }\end{array}$ & $\begin{array}{l}\text { L'ensemble des activités, } \\
\text { des moyens planifiés pour } \\
\text { développer les applica- } \\
\text { tions du nouveau système }\end{array}$ & $\begin{array}{l}\text { Description du plan de } \\
\text { développement, des per- } \\
\text { sonnes engagées dans le } \\
\text { projet et du moment de } \\
\text { préparation }\end{array}$ \\
\hline $\begin{array}{l}\text { B. Engagement de spécia- } \\
\text { listes }\end{array}$ & $\begin{array}{l}\text { Décisions de l'organisa- } \\
\text { tion qui doit déterminer à } \\
\text { quelle étape intervient le } \\
\text { perrsonnel en infor- } \\
\text { matique et les consultants } \\
\text { de firmes privées }\end{array}$ & $\begin{array}{l}\text { Description du rôle et du } \\
\text { moment où sont in- } \\
\text { tervenus le personnel et } \\
\text { les spécialistes du secteur } \\
\text { privé }\end{array}$ \\
\hline $\begin{array}{l}\text { C. Définition de politiques } \\
\text { de confidentialité et de } \\
\text { mécanismes de sécurité }\end{array}$ & $\begin{array}{l}\text { Politiques assurant la con- } \\
\text { fidentialité des informa- } \\
\text { tions et établissement de } \\
\text { procédures concernant la } \\
\text { collecte, l'enregistrement } \\
\text { et l'utilisation de l'infor- } \\
\text { mation }\end{array}$ & $\begin{array}{l}\text { Description des politiques } \\
\text { et des mécanismes, du } \\
\text { moment d'élaboration et } \\
\text { des personnes concernées }\end{array}$ \\
\hline $\begin{array}{l}\text { D. Planification du budget } \\
\text { Établir si l'on dispose des } \\
\text { ressources techniques et } \\
\text { économiques suffisantes } \\
\text { pour l'informatisation }\end{array}$ & $\begin{array}{l}\text { Description des instances } \\
\text { qui financent le projet et } \\
\text { du moment où est planifié } \\
\text { le budget }\end{array}$ \\
\hline
\end{tabular}

La phase d'implantation se cristallise autour de deux activités principales. La mise en place du système débute par la réception et I'installation du matériel. Les indicateurs de cette activité sont la description du moment et de l'endroit où est installé le matériel informatique, l'assistance technique offerte et la formation donnée au personnel. La deuxième activité de la phase d'implantation est la mise en fonction du nouveau système et le suivi de son fonctionnement, suivi qui amène des changements au système et des ajustements de la part de l'organisation incluant des évaluations périodiques du système. Les indicateurs sont : le moment où le système est utilisé, les changements ou modifications apportés et les ajustements de l'organisation. 
À l'étape de la phase III dite de clarification se précisent de façon plus permanente les modes d'utilisation et les applications du système. Elle décrit la mise en fonction du système à la suite des modifications et des ajustements, les applications subséquentes, de même que les dispositions prises pour l'utilisation de l'ordinateur.

La phase IV, appelée phase de routine, est celle où l'ordinateur est maintenant incorporé dans les activités de l'organisation et n'est plus considéré comme une innovation. L'utilisation du système est devenue opérationnelle et ses applications plus régulières et mieux intégrées au milieu. À mesure que les cliniciens utilisent I'informatique, de nouvelles applications et utilisations se font jour.

\section{La collecte et l'analyse des données}

L'étude du processus d'implantation portait sur quatre cliniques pédiatriques. Pour préserver la confidentialité, ces cliniques ont été désignées sous le vocable de clinique A, B, C ou D. Les cliniques $A$ et $B$ sont celles qui ont été informatisées les premières, alors que les cliniques $C$ et $D$ ont amorcé le processus d'implantation au cours de la recherche.

La clinique $A$ assure la surveillance de près de 300 enfants atteints d'une maladie chronique. Ces derniers demandent des soins à long terme et un suivi médical régulier : tests de sang, surveillance du régime alimentaire, du poids, des médicaments. Quatre visites annuelles sont prévues au protocole de soins établi pour chaque enfant. La clinique $B$, pour sa part, s'occupe des enfants porteurs d'une malformation. Une semaine après la naissance, ces enfants sont dirigés vers la clinique et sont hospitalisés au cours de la première année deux ou trois fois suivant les chirurgies nécessaires. La clinique $B$ intervient auprès de 190 enfants, dont une cinquantaine de nouveaux cas chaque année. La clinique $C$ s'occupe d'enfants atteints d'une maladie grave nécessitant des soins spécialisés et des traitements intensifs et palliatifs. Près de 1500 enfants et familles sont suivis à cette clinique. Quant à la clinique $D$, elle s'occupe d'enfants et de mères infestés par un virus pouvant conduire à différentes formes d'infections. Près de 40 enfants et leurs mères sont suivis à la clinique. De plus en plus, les soins sont donnés en externe, diminuant ainsi les hospitalisations.

Dans le texte, nous parlons de "cliniques informatisées" lorsque nous faisons référence aux cliniques $A$ et $B$ et de « cliniques en voie $d^{\prime}$ informatisation " lorsque nous faisons allusion aux cliniques $C$ et $D$. Pour recueillir les données touchant l'implantation 
de l'informatique, deux instruments de collecte de données ont été utilisés, soit l'étude documentaire et l'entrevue semi-dirigée. Les documents concernant le projet informatique que possédaient les intervenantes sociales des cliniques informatisées et le gestionnaire ${ }^{2}$ du service social ont été consultés dans le but d'y recueillir toute l'information disponible. Nous avons par la suite réalisé des

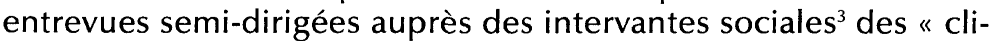
niques informatisées" et du gestionnaire du service social. La même procédure a été utilisée pour les " cliniques en voie d'informatisation » : consultation des dossiers du service social, de ceux des intervenantes des cliniques et entrevues semi-dirigées avec celles-ci. La collecte des données s'est réalisée à trois moments différents, comme on peut l'observer au tableau 3.

Contrairement à nos prévisions initiales, l'étude documentaire n'a pu être effectuée dans les cliniques $C$ et $D$ à notre dernière collecte (hiver 1991). En effet, le processus d'informatisation a été interrompu dans ces deux cliniques peu après notre deuxième collecte de données.

Nous appuyant sur la théorie de Yin (1984), nous avons choisi comme stratégie générale d'analyse le développement d'un cadre général de description de cas. L'analyse elle-même du processus d'implantation s'est faite en plusieurs étapes. La première étape relève de l'analyse chronologique. Elle consiste à mettre tous les événements associés à l'implantation dans un ordre strictement chronologique. Cette étape assez longue et descriptive n'est pas reproduite dans le présent article.

\section{TABLEAU 3}

\section{Moments de collecte des données concernant l'implantation}

\begin{tabular}{|c|c|c|}
\hline Collecte H-89 & Collecte H-90 & Collecte H-91 \\
\hline $\begin{array}{l}\text { Cliniques } A, B \text { : } \\
1^{\text {re étude documentaire. }}\end{array}$ & $\begin{array}{l}\text { Cliniques A, B : } \\
1^{\text {res }} \text { entrevues avec les in- } \\
\text { tervenantes sociales et un } \\
\text { gestionnaire du service } \\
\text { social. }\end{array}$ & $\begin{array}{l}\text { Cliniques A, B : } \\
2^{\mathrm{e}} \text { étude documentaire; } \\
2^{\text {es }} \text { entrevues avec les in- } \\
\text { tervenantes sociales. }\end{array}$ \\
\hline $\begin{array}{l}\text { Entrevue avec un gestion- } \\
\text { naire de l'hôpital. }\end{array}$ & $\begin{array}{l}\text { Cliniques C, D : } \\
1^{\text {re étude documentaire; }} \\
1^{\text {res }} \text { entrevues avec les in- } \\
\text { tervenantes sociales. }\end{array}$ & \\
\hline
\end{tabular}


La seconde étape relève à la fois de l'analyse chronologique et de la comparaison des faits à la théorie (pattern-matching). Il s'agissait de procéder au classement des événements dans les activités propres à chacune des phases. Certaines activités à l'intérieur de ces phases et certaines phases du processus d'implantation pouvaient ne pas se retrouver dans les observations recueillies ou avoir eu lieu à des moments différents de nos prévisions originales. Nous pouvions aussi relever de nouvelles activités dans chacune des phases d'implaritation, activités qui n'étaient pas décrites dans le modèle de base, ou encore en arriver à un ordre différent de réalisation des activités à l'intérieur de chaque phase.

Les résultats de cette deuxième étape dite de l'analyse chronologique par phase ont été présentés sous forme de tableaux où figurent tous les événements réalisés durant le processus d'implantation de chacune des quatre cliniques (Béliveau, Poulin, Beaudoin, 1991). Cela nous permet d'avoir une vision claire de l'ensemble des activités dans le temps, d'en connaître le déroulement et de susciter une réflexion sur la logique des événements. Dans cet article, nous présentons des formes adaptées des tableaux originaux qui figurent dans le rapport de recherche. Ainsi, nous avons choisi d'étudier plus en détail les activités de la phase I en les classifiant selon le moment où elles débutent dans chacune des quatre cliniques. Les tableaux 4 et 5 ne reproduisent cependant pas le nombre de fois que chaque activité a eu lieu : une activité peut s'être répétée à plusieurs reprises et à différents moments. Nous analyserons d'abord les données recueillies dans les cliniques $A$ et $B$, puis suivra I'analyse des activités des cliniques $C$ et $D$.

La troisième étape d'analyse consiste à construire un modèle explicatif (explanation-building) de la réalité qui met en relief les ressemblances et les différences avec notre typologie de départ et fournit l'explication du "comment » et du "pourquoi » des phénomènes observés. Le modèle d'implantation résulte de cette étape d'analyse et est reproduit séparément dans ce texte pour les cliniques $A$ et $B$ et pour les cliniques $C$ et $D$. Cette façon de faire permet de présenter distinctement le cheminement adopté par chaque groupe de cliniques.

Une quatrième étape d'analyse tente d'établir les interrelations entre les résultats obtenus d'une clinique à l'autre en retenant leurs similitudes et leurs différences (cross-case analysis). Cette analyse comparative est effectuée entre les cliniques rendues à un degré d'informatisation similaire (clinique A vs clinique B et clinique $C$ vs clinique $D$ ) et entre le groupe des cliniques informatisées (cliniques A et B) et le groupe des cliniques en voie d'informatisation (cliniques $C$ et $D$ ). Cette dernière analyse comparative nous 
TABLEAU 4

Début des activités de la phase I par ordre chronologique ${ }^{4}$

\begin{tabular}{|c|c|c|}
\hline & Clinique A & Clinique B \\
\hline Juin 1978 & & $\begin{array}{c}\text { Activité } 1: \text { Mise en route du } \\
\text { projet }\end{array}$ \\
\hline $\begin{array}{l}\text { Janvier-février } 1981 \\
\text { Décembre } 1981\end{array}$ & & $\begin{array}{c}\text { Activité } 2 \text { : Définition des } \\
\text { besoins } \\
\text { Activité D : Budget }\end{array}$ \\
\hline Janvier 1982 & $\begin{array}{c}\text { Activité } 1: \text { Mise en route du } \\
\text { : projet }\end{array}$ & \\
\hline $\begin{array}{l}\text { Septembre } 1983 \\
\text { Octobre } 1983 \\
\text { Décembre } 1983\end{array}$ & $\begin{array}{l}\text { Activité } 4 \text { : Collecte d'infor- } \\
\text { mations } \\
\text { Activité } 5 \text { : } \begin{array}{l}\text { Conception du } \\
\text { système }\end{array}\end{array}$ & $\begin{array}{c}\text { Activité } 5: \text { Conception du } \\
\text { système }\end{array}$ \\
\hline $\begin{array}{l}\text { Janvier } 1984 \\
\text { Juin } 1984 \\
\text { Août } 1984 \\
\text { Novembre } 1984\end{array}$ & 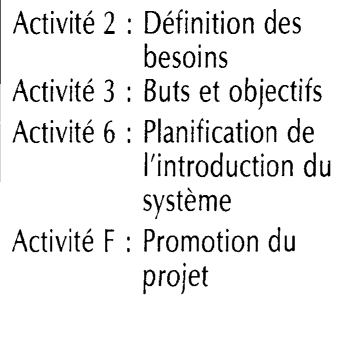 & Activité 3 : Buts et objectifs \\
\hline Octobre 1985 & & $\begin{array}{c}\text { Activité } \mathrm{F} \text { : Promotion du } \\
\text { projet }\end{array}$ \\
\hline $\begin{array}{l}\text { Mars } 1986 \\
\text { Mai } 1986 \\
\text { Novembre } 1986\end{array}$ & $\begin{array}{c}\text { Activité B : Engagement de } \\
\text { spécialistes } \\
\text { Activité D : Budget } \\
\text { Activité E : Droits d'auteur }\end{array}$ & $\begin{array}{l}\text { Activité B : Engagement de } \\
\text { spécialistes } \\
\text { Activité } 6 \text { : } \begin{aligned} \text { Planification de } \\
\text { l'introduction du } \\
\text { système }\end{aligned} \\
\text { Activité E : Droits d'auteur }\end{array}$ \\
\hline Septembre 1987 & $\begin{array}{c}\text { Activité A : Plan de dévelop- } \\
\text { pement }\end{array}$ & \\
\hline Février 1988 & & $\begin{array}{l}\text { Activité A : Plan de dévelop- } \\
\text { pement }\end{array}$ \\
\hline
\end{tabular}




\section{TABLEAU 5}

Début des activités de la phase I par ordre chronologique

\begin{tabular}{|c|c|c|}
\hline & Clinique $\mathrm{C}$ & Clinique D \\
\hline Janvier 1989 & $\begin{array}{l}\text { Activité 1: } \begin{array}{l}\text { Mise en route du } \\
\text { projet }\end{array}\end{array}$ & $\begin{array}{cl}\text { Activité } 1: & \text { Mise en route du } \\
& \text { projet } \\
\text { Activité D : Planification du } & \begin{array}{l}\text { Pladget } \\
\text { budgen }\end{array}\end{array}$ \\
\hline Septembre 1989 & $\begin{array}{c}\text { Activité D : Planification du } \\
\text { budget }\end{array}$ & \\
\hline Novembre 1989 & & $\begin{aligned} \text { Activité 6: } & \text { Planification de } \\
& \text { l'introduction du } \\
& \text { système }\end{aligned}$ \\
\hline Janvier 1990 & $\begin{aligned} \text { Activité 6: } & \text { Planification de } \\
& \text { l'introduction du } \\
& \text { système }\end{aligned}$ & Activité 3: Buts et objectifs \\
\hline Février 1990 & $\begin{array}{c}\text { Activité A : Plan de dévelop- } \\
\text { pement }\end{array}$ & \\
\hline Avril 1990 & $\begin{array}{l}\text { Activité } 2 \text { : Définition des } \\
\text { besoins } \\
\text { Activité 3: Buts et objectifs }\end{array}$ & 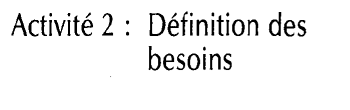 \\
\hline
\end{tabular}

amène à reprendre le processus d'implantation et à présenter un modèle modifié de processus d'informatisation dont les organismes et les établissements de service social pourraient s'inspirer. $C^{\prime}$ est ce que nous faisons ressortir dans les paragraphes intitulés "Que retenir de cette expérience?».

\section{L'analyse chronologique par phases : cliniques A et B}

Notre hypothèse de départ voulait que chacune des phases se développe à la suite l'une de l'autre selon un ordre logique. Parmi les quatre phases du processus, trois étaient réalisées dans les cliniques $A$ et $B$, à des degrés divers, au moment de la dernière collecte. À la clinique $A$, la phase préparatoire a débuté en janvier 1982 par la " mise en route du projet ". L'implantation s'est amorcée en mai 1984, soit un peu plus de deux ans plus tard. Par la suite, les activités des deux phases se sont poursuivies en parallèle. Il y a donc eu antériorité de la phase I sur la seconde au départ, puis action parallèle pendant le reste de la période, soit de mai 1984 à février 1991. La phase de clarification, où se précisent de façon plus 
permanente les modes d'utilisation et les applications du système, a commencé en 1990 à la clinique A. Selon nous, la clinique A n'était pas tout à fait rendue à la phase de routine.

Pour ce qui est de la clinique $B$, la phase préparatoire a débuté en juin 1978. La seconde phase, celle de l'implantation, s'est amorcée en septembre 1987, soit neuf ans plus tard. Par la suite, les activités des deux premières phases se sont déroulées parallèlement. Il y a donc eu antériorité de la phase I sur la seconde pour le début des activités, puis action parallèle pendant le reste de la période, soit de septembre 1987 à février 1991. La phase de clarification a commencé, tout comme à la clinique $A$, en 1990.

Les données recueillies dans les cliniques $A$ et $B$ nous ont amenés à reconnaître et à introduire deux nouvelles activités dans la phase I. Ces activités sont désignées sous les titres « Reconnaissance des droits d'auteur » et "Promotion du projet » (activités E et $\mathrm{F}$ respectivement).

Par reconnaissance des droits d'auteur, nous entendons « I'action entreprise par les intervenantes sociales des cliniques A et B pour se faire reconnaître formellement le droit d'exploiter à leur compte (du moins partiellement) le logiciel d'intervention familiale et sociale ". La seconde activité que nous avons ajoutée porte le nom de " promotion du projet » et se définit comme " l'ensemble des techniques et des moyens utilisés pour promouvoir le projet et son produit, dans le but d'assurer son développement et de partager l'expérience acquise avec d'autres personnes intéressées".

Pour chacune des cliniques, nous avons replacé les différentes activités de la phase I en fonction du moment de leur apparition. Le tableau 4 les présente pour les cliniques $\mathrm{A}$ et $\mathrm{B}$.

Les activités numérotées étaient, selon le modèle initial, placées dans l'ordre logique et devaient se dérouler idéalement selon cet ordre. On peut constater qu'à la clinique A, les activités 2, 3, 4 et 5 ont lieu dans un ordre différent de celui présenté dans le modèle initial. La mise en route des activités se fait par grappes. Bien que cela n'apparaisse pas au tableau 4, les données recueillies nous permettent de dire que la réalisation de la plupart de ces activités est concentrée en 1986 et 1987. L'activité C (Définition de politiques de confidentialité) est absente du tableau 4 puisque aucune date précise n'a permis de retracer le début de sa réalisation. Par rapport au modèle de référence, les activités 3 et 5 ont débuté dans un ordre chronologique inversé. Dans les deux cliniques, on a conçu le système avant d'établir les buts et les objectifs. Dans la clinique $B$ la mise en route des activités classées de 1 à 6 se fait aussi par grappes et la réalisation de la plupart de ces activités est concentrée principalement en 1986 et 1987, alors que l'initiative 
du projet remonte à 1978. Notons que dans le cas des activités $E$ (droits d'auteur) et F (promotion du projet), il n'y a aucun lien entre ces activités et la séquence temporelle de la première phase.

\section{Modèle d'implantation propre aux cliniques A et B}

Bien que nous n'ayons présenté dans cet article que l'analyse chronologique des activités de la phase I, nous faisons ressortir ici les principales observations concernant les trois premières phases du processus d'implantation réalisé dans les cliniques informatisées. Ce processus, rappelons-le, visait à informatiser les données du service social et à les intégrer aux dossiers médicaux des cliniques.

Les modèles d'implantation suivis par les cliniques A et B comportent plusieurs similitudes. La démarche suivie dans ces cliniques couvre trois des quatre phases du modèle initial : la phase préparatoire, la phase d'implantation et la phase de clarification. Seule la phase de routine n'est pas amorcée. On y retrouve également la quasi-totalité des activités prévues et identifiées dans les phases préparatoire et d'implantation. La seule exception concerne l'activité 4 (collecte d'informations) que l'on ne retrouve pas dans le cheminement de la clinique $B$ puisque cette activité a été entreprise à la clinique $A$ seulement, pour le compte des deux cliniques. Nous avons également relevé pour ces mêmes cliniques deux activités non prévues et prenant place à la phase préparatoire. Il s'agit de la reconnaissance des droits d'auteur (activité E) et de la promotion du projet (activité F). En ce qui concerne la phase préparatoire nous avons vu que plusieurs activités doivent se dérouler idéalement selon un certain ordre. Pour d'autres, l'aspect chronologique semble moins important. Dans les deux cliniques, I'ordre d'apparition des phases I, II et III a été respecté, du moins en ce qui concerne leur point de départ.

Par ailleurs, en ce qui a trait au moment d'apparition des activités de la phase I dans la clinique $A$, on a pu constater que la séquence des activités a peu de liens chronologiques avec le modèle proposé et repose sur une logique différente, sauf pour le début et la fin de la phase I : la mise en route du projet apparaît au début du processus et la planification de l'introduction de l'informatique (mai, juin 1984) se réalise à la même époque que le début de la phase d'implantation (phase II). Les événements peuvent alors être précédés ou suivis indifféremment par d'autres s'il n'y a pas de lien direct de cause à effet. Une autre explication résiderait dans le fait qu'aucun canevas de travail et de développement n'a été ou n'a pu être élaboré au début du projet. Les démarches 
semblent s'être accomplies au fur et à mesure des besoins. Le processus n'obéit pas à une démarche logique, ordonnée et planifiée par une autorité supérieure en vue de son implantation dans toute l'organisation ou dans une partie seulement. II s'agit d'un processus entrepris par le bas. L'importance des démarches réalisées par l'intervenante de la clinique dans tout le processus en témoigne.

On se trouve donc en présence d'un processus d'informatisation éclaté, c'est-à-dire que certaines activités ont été menées simultanément sans qu'elles apparaissent dans l'ordre logique proposé dans le schéma de départ. Nous avons pu observer, au cours de la recherche, que la plupart des activités du processus de la clinique $A$ se sont poursuivies simultanément, de façon ponctuelle, avec des temps morts et des périodes actives (1984, 1988 et 1989) ou très intensives (1986 et 1987) ${ }^{4}$. Le projet évolue par bonds ou en spirale et en discontinuité, de sorte que les activités de la phase préparatoire s'échelonnent sur une période de neuf ans.

Certaines explications avancées dans le cas de la clinique $A$ peuvent s'appliquer assez bien à la clinique $B$. À première vue, la séquence des activités amorcées pendant la phase I semble avoir un certain lien logique si l'on tient compte du fait que les activités 1 , 2 et 6 , soit trois activités sur cinq, débutent selon l'ordre chronologique prévu. Le fait que la collecte d'informations (activité 4) soit absente de la clinique $B$ et qu'il y ait un délai de huit années entre le début de la première activité (mise en route du projet) et la planification de l'introduction du système (activité 6) est pour le moins surprenant. Un plan de travail ou de développement de l'informatisation n'a pas été conçu au début du projet. Ainsi, plusieurs démarches de la clinique $B$ découlent des initiatives prises dans la clinique $A$. C'est le cas des activités 5 (conception du système), $B$ (engagement de spécialistes) et $E$ (droits d'auteur). On peut aussi s'étonner qu'on se soit préoccupé des buts et objectifs (activité 3) après la conception du système (activité 5 ). Pour ce qui est de l'activité 4 (collecte d'informations), il semblerait que la clinique B ait bénéficié des acquis de la clinique $A$.

Les particularités du processus suivi dans les deux cliniques font ressortir plusieurs ressemblances. Ainsi, dans les deux cas, l'initiative du projet vient de l'intervenante sociale et non de la direction de l'hôpital ou du CSS ou du service social lui-même. Cela ne va pas sans perturber le fonctionnement des deux établissements : le développement de l'informatisation des cliniques, d'une part, et de l'informatisation des données du service social, d'autre part, n'était pas prévu si tôt, ni considéré comme prioritaire aussi bien par l'hôpital que par le CSS. La double appartenance 
structurelle des cliniques et la mise en route du projet par la base de la hiérarchie de l'organisation entraînent plusieurs conséquences : les intervenantes ont voulu faire reconnaître leurs droits d'auteur pour bien faire valoir leur contribution au projet; le mode d'obtention des crédits et la façon de promouvoir le projet et de prendre les décisions ont été largement dictés par ces conditions organisationnelles d'implantation du processus d'informatisation.

Plusieurs activités, bien qu'elles soient indiquées dans le processus d'implantation des cliniques $A$ et $B$, se sont déroulées dans une séquence différente de celle suggérée dans les écrits sur le sujet. Nous faisons référence ici aux analyses liées à la planification et à l'évaluation des activités. L'absence de telles analyses, que nous attribuons principalement au fait que les intervenantes $n^{\prime}$ ont pas de budget, qu'elles sont peu expérimentées en matière de planification et que I'hôpital et le CSS sont pris au dépourvu par le projet, peut expliquer en partie la faiblesse du modèle d'implantation suivi, qui se traduit par un manque de cohérence entre les différentes activités.

Signalons également la difficulté d'étendre l'innovation aux autres cliniques, car les instigatrices du projet n'ont aucune autorité sur le service social et les autres cliniques. Elles ont seulement un pouvoir de persuasion. Les membres du comité informatique, dont les intervenantes des cliniques $\mathrm{A}$ et $\mathrm{B}$ font partie, ont plus d'une fois demandé au CSS que leur projet soit reconnu comme projet-pilote; mais ils n'ont pu obtenir ce statut, ce qui aurait conféré un caractère institutionnel au projet. L'approche par projet-pilote suppose la réalisation d'un projet informatique dans une ou quelques unités administratives; après analyse et évaluation, le reste de l'organisation est informatisé graduellement. On ne peut qualifier le processus suivi d'approche par projet-pilote, car l'appui institutionnel n'était pas suffisant et n'a pas permis de faire l'analyse et l'évaluation appropriées. Le service social s'est toutefois engagé à soutenir l'effort d'informatisation et a reconnu le travail de ses intervenantes, en acceptant par la suite, comme rapport de révision, le rapport informatisé élaboré au cours du processus $\mathrm{d}^{\prime}$ informatisation des cliniques $\mathrm{A}$ et B. L'organisation a aussi favorisé l'utilisation de la grille $d^{\prime}$ 'intervention familiale et sociale dans les autres cliniques.

En résumé, les particularités du processus d'informatisation dans les cliniques $A$ et $B$ reposent sur le fait qu'il ne prend pas sa source chez les gestionnaires des institutions et que le service social a une double allégeance. Cela aurait influencé de nombreuses démarches et décisions tout au long de ce projet, et amené de l'incohérence dans la coordination des différentes activités. Ces 
conditions ont eu comme conséquence d'entraîner de nombreux délais dans les décisions aux différents stades de développement du processus. On peut penser que la planification des activités aurait été un élément facilitant la progression du processus et aurait pu diminuer, voire éviter les nombreuses difficultés et embûches rencontrées. La résistance à un changement peut fort probablement expliquer une bonne part des difficultés liées à l'informatisation des données du service social. Les instigatrices du projet ont eu à sensibiliser la direction des deux institutions et ont mobilisé de nombreuses énergies à cet égard. La résistance rencontrée semblerait provenir de tous les niveaux hiérarchiques ainsi que des pairs. Les motifs de résistance peuvent évidemment différer selon le niveau hiérarchique : d'une part, cela vient perturber les priorités des gestionnaires; d'autre part, on craint de ne pouvoir étendre l'information des données en raison du peu d'intérêt manifesté par certaines intervenantes des autres cliniques.

\section{L'analyse chronologique par phases : cliniques C et D}

Les résultats concernant la clinique $C$ démontrent que les deux premières phases sont réalisées partiellement. Ainsi, la phase préparatoire a débuté en janvier 1989, alors que la seconde, celle de l'implantation, a été amorcée en octobre de la même année. Par la suite, les activités des deux phases se sont poursuivies de façon parallèle. Les observations recueillies à la clinique $D$ démontrent que la première phase seulement a été amorcée (la mise en route du projet). Le processus d'implantation n'était pas très avancé dans ces deux cliniques puisque le projet d'informatisation a été mis en veilleuse au cours de l'année 1990.

Comme on peut l'observer au tableau 5, seulement six des activités prévues à la phase préparatoire ont été amorcées à la clinique $C$. On peut s'étonner en regardant le même tableau que I'on ait entrepris la planification de l'introduction du système avant de définir les besoins et de déterminer les buts. Quant à la clinique $D$, le nombre des activités amorcées n'est que de cinq et présente un ordre différent de celui prévu dans notre modèle initial.

\section{Modèle d'implantation propre aux cliniques $\mathbf{C}$ et $\mathbf{D}$}

Il est difficile, à ce stade d'informatisation, de saisir la logique $d^{\prime}$ implantation à la clinique $C$. Cependant, les efforts sont mis surtout à faire l'acquisition de matériel informatique par des de- 
mandes de subvention, car la planification des budgets (activité D) commence dès septembre 1989, soit immédiatement après la première activité de la première phase.

Comme à la clinique $C$, peu d'activités ont été réalisées à la clinique $\mathrm{D}$. Les premières activités de mise en route du projet en janvier 1989 sont immédiatement suivies de la planification du budget (activité D). Les trois autres activités commencent quelques mois plus tard. On observe que les activités de la clinique D n'apparaissent pas dans l'ordre prévu dans le modèle initial. Il est pour le moins étonnant de constater que la définition des besoins (activité 2) intervient juste avant que le processus soit interrompu. Normalement, cette activité doit intervenir bien avant les autres afin d'orienter les démarches qui devraient suivre. Amorçant le processus d'informatisation au moment où les étapes préliminaires avaient été réalisées dans les autres cliniques au cours des dernières années, la clinique $\mathrm{D}$ importe en bloc le système développé ailleurs et centre son attention sur l'obtention des fonds nécessaires à la réalisation du projet pour ensuite passer rapidement à l'introduction du système (activité 6).

La démarche d'implantation suivie par les cliniques en voie $d^{\prime}$ informatisation comporte plusieurs ressemblances. Au moment de notre collecte de données à l'hiver de 1990 le processus d'implantation n'en était qu'à ses débuts dans les deux cliniques. À la clinique $C$, les phases I et II sont amorcées, mais partiellement. La clinique $D$, pour sa part, n'a pas entamé la deuxième phase, et la première, celle de la préparation, n'est pas très avancée, tout comme à la clinique $\mathrm{C}$. Dans une clinique comme dans l'autre plusieurs activités de la phase préparatoire n'ont pas encore débuté.

Bien qu'à l'intérieur des cliniques en voie d'informatisation les instigatrices du projet soient les intervenantes sociales de ces cliniques, il n'en reste pas moins que l'idée originale du projet vient des intervenantes des cliniques déjà informatisées (A et $B$ ) qui appuient les démarches de leurs collègues. Dans les cliniques $C$ et $D$, le projet d'informatisation des données de service social a été mis en veilleuse. Plusieurs explications peuvent être avancées. Par exemple, le système proposé ne coïncidait peut-être pas exactement avec les besoins de ces cliniques, en particulier ceux de la clinique $\mathrm{D}$. On pourrait aussi alléguer que tous les membres de ces cliniques n'ont pas endossé totalement le projet d'informatisation. On peut croire que certaines activités ont été entreprises trop tôt et de façon hâtive avant que les objectifs n'aient été précisés et que les activités n'aient été planifiées de façon à satisfaire les principaux intéressés. Aussi, le manque de ressources (absence de sub- 
ventions) pour ces deux cliniques a entraîné une démobilisation et une perte d'énergies conduisant à l'interruption des initiatives des intervenantes. Une des principales raisons de cette interruption des activités ne serait-elle pas justement reliée au mode de mise en route du projet ? L'initiative provient certes des intervenantes des cliniques $\mathrm{C}$ et $\mathrm{D}$, mais l'idée vient de membres extérieurs à la clinique et n'est pas soutenue par un projet institutionnel. La motivation et la persévérance dans la poursuite du projet sont dès lors fort différentes. On ne peut s'étonner dans ce contexte qu'il n'ait pas survécu.

\section{Que retenir de cette expérience?}

Dans un premier temps, nous comparons les particularités du processus d'implantation réalisé dans les cliniques informatisées et chez celles en voie d'informatisation. Cela nous amènera à formuler quelques propositions pour faciliter l'implantation de l'informatique et à suggérer un modèle d'implantation révisé.

Le modèle d'implantation suivi par les cliniques informatisées et en voie d'informatisation comporte des similitudes, mais aussi des différences. Au premier abord, si l'on s'en tient au modèle, on observe que le processus est beaucoup plus avancé et développé dans les cliniques informatisées que dans celles en voie d'informatisation. Cela n'est pas étonnant si l'on considère que dans les cliniques A et B la mise en route du projet remonte à 1982 et 1978 respectivement, comparativement à 1989 dans les cas des cliniques $C$ et $D$. Pour ce qui est de la démarche d'implantation, on observe plusieurs similitudes entre les cliniques informatisées et en voie d'informatisation, surtout en ce qui a trait à l'enclenchement du processus et au mode de financement. Cependant, comparativement aux cliniques $A$ et $B$, plusieurs activités de la phase préparatoire ne devaient pas avoir lieu dans les cliniques $C$ et $D$. Ainsi, on prévoyait dans ces dernières cliniques transposer avec quelques adaptations le système déjà développé dans les cliniques informatisées. Cependant, on peut se demander si le modèle des cliniques A et B convenait aux cliniques en voie d'informatisation. En d'autres termes, aurait-il été préférable de s'attarder davantage à la définition des besoins, à la précision des buts et des objectifs, de refaire l'exercice de collecte d'informations et d'adapter le logiciel aux besoins ? De plus, on note que les efforts des cliniques en voie $d^{\prime}$ informatisation se sont portés très tôt vers la recherche de fonds, ces cliniques n'ayant pas procédé au préalable à la collecte d'informations (activité 4) et à la conception du système (activité 5). 
Pour ce qui est des similitudes, la principale concerne sans nul doute les personnes qui ont lancé l'idée du projet. Dans toutes les cliniques, ce sont les intervenantes sociales qui sont les éléments moteurs du processus. Par contre, dans le cas des cliniques en voie d'informatisation, l'idée du projet prend sa source chez des membres extérieurs à ces cliniques, les intervenantes des cliniques déjà informatisées. On se souvient que dans ces dernières cliniques, les intervenantes étaient soutenues par tous les membres de l'équipe multidisciplinaire puisqu'on procédait à l'informatisation complète des données médicales et psychosociales. Par contre, dans les cliniques C et D, l'idée d'informatiser les données psychosociales n'entrait pas dans le projet de l'une ou l'autre clinique et n'était pas née du désir de l'ensemble des membres. Finalement, alors que les cliniques $A$ et $B$ ont à peu près franchi les trois premières phases et se dirigent vers un mode d'application somme toute bien intégré et fonctionnel, les cliniques $C$ et $D$ quant à elles ont mis en veilleuse le projet d'informatisation alors qu'elles entraient à peine dans la deuxième phase. Plusieurs raisons pouvant expliquer ce fait ont été avancées antérieurement.

Il va sans dire qu'un projet d'une telle ampleur a nécessité de la part des intervenantes sociales et des membres du service social des efforts considérables, si l'on tient compte plus particulièrement du contexte dans lequel il s'est développé. Le projet, et ce dans toutes les cliniques, prend sa source à la base de la hiérarchie, contrairement à ce qui se réalise habituellement lorsque l'on songe à amorcer un projet d'informatisation. De plus, la double allégeance du service social à la fois à l'hôpital et au CSS, comme nous l'avons vu, n'a pas facilité les choses. Dans les cas des cliniques A et $B$, où le processus est très avancé, on remarque que les activités ont été menées sans plan détaillé et sans évaluation, comme on le suggère dans la plupart des modèles théoriques. Cela se reflète par un manque de synchronisation des différentes étapes. Malgré tout, on a pu observer que le processus suivi correspond en grande partie aux phases et aux activités, comme le prévoyait le modèle théorique. La logique d'enchaînement des activités à l'intérieur des phases n'est cependant pas toujours respectée.

L'analyse des observations suggère que la planification et l'évaluation des activités constituent des étapes essentielles du processus. Elles permettent de faire progresser l'action et aident à éviter de nombreux écueils, et surtout des délais considérables dans la prise de décision. Ces délais se sont révélés parfois démobilisants à bien des égards et ont entraîné une perte d'énergie, entre autres en orientant cette énergie à des fins de promotion du projet à l'extérieur de l'organisation. Il est nécessaire de s'appuyer 
sur un modèle $d^{\prime}$ implantation permettant une bonne planification du processus.

Gandy et Tepperman (1990) concluaient dans leur ouvrage qu'on ne peut surestimer l'importance de la planification et qu'en dépit de la conscience que l'on en a plusieurs organisations ne planifient pas l'introduction de l'ordinateur dans leurs services. Selon ces auteurs, de façon générale peu de ressources financières sont prévues et allouées à l'entraînement des usagers ou à l'engagement de techniciens pour superviser de façon continue l'utilisation du matériel informatique. Dans le cadre des cliniques, le processus d'informatisation des données de services social et leur intégration aux données médicales n'étaient ni prévus ni pris en charge officiellement par les autorités des deux établissements (CSS et hôpital). Dans un tel contexte, les problèmes de planification et d'allocation de ressources ont été vécus de façon encore plus aiguë. Les instigatrices du projet ont eu à tenir plusieurs rôles, habituellement dévolus aux gestionnaires et aux techniciens responsables de la bonne marche d'un projet d'informatisation.

À partir de ce qui précède, il appert que le modèle initialement élaboré en quatre phases est valable comme modèle à suivre pour l'implantation d'un système d'informatique en service social. Les activités à l'intérieur des phases semblent appropriées et celles de la phase I ont été bien testées. Cependant, les activités de cette phase auraient avantage à se dérouler selon une autre logique que nous proposons à la place du modèle initial.

\section{Modèle initial}

Mise en route du projet

Définition des besoins

Détermination des buts et objectifs

Collecte d'informations

Conception du système

Planification de l'introduction du système

Plan de développement du système

Engagement de spécialistes en informatique

Définition de politiques de confidentialité

Planification du budget
Modèle proposé

Mise en route du projet

Définition des besoins

Détermination des buts et objectifs

Collecte d'informations

Planification du budget

Engagement de spécialistes en informatique

Définition de politiques de confidentialité

Conception du système

Planification de l'introduction du système 
À la phase II, en plus des activités déjà mentionnées dans le tableau initial, on devrait ajouter une autre activité dite d'évaluation. Les activités de la phase II pourraient donc se lire comme suit :

1. Mise en place du système : installation

2. Formation du personnel et assistance technique

3. Mise en fonction et suivi du système

4. Évaluation

5. Modification du système

6. Ajustement de l'organisme

L'activité A, "Préparation d'un plan de développement du système ", qu'on trouvait à la phase I, aurait mieux sa place dans la quatrième phase, soit à la phase de routine. Ainsi qu'il a été défini antérieurement, cette activité vise à développer les applications du nouveau système et suppose qu'on reprenne les étapes à partir de la phase I. On se retrouve donc au début du processus qui consiste à mettre en branle de nouveaux projets d'informatisation.

On ne peut faire autrement qu'apprécier l'esprit d'initiative et la ténacité des intervenantes sociales qui ont contribué à faire avancer la réflexion et les connaissances en matière $d^{\prime}$ utilisation de l'ordinateur à des fins d'intervention clinique. Nous espérons que cette expérience ouvrira la voie à d'autres innovations. Par ailleurs, les observations recueillies tendent à remettre en question le processus suivi à partir de la seule initiative de membres de la base de l'organisation. La réussite de l'implantation d'une innovation technologique comme celle de l'informatisation des données du service social requiert l'action concertée de l'ensemble de l'organisation, tant de la part des gestionnaires que des intervenantes de la base.

Enfin, ces observations permettent, une fois de plus, de constater que le phénomène de résistance au changement est présent tant chez les praticiennes que chez les gestionnaires. Cette réflexion nous renvoie aux constatations de l'étude de Béliveau et Poulin (1987) sur les perceptions et les attitudes des intervenants sociaux.

\section{Notes}

1. Dans les pages qui suivent, le masculin est employé comme générique, sans discrimination et à la seule fin d'alléger le texte.

2. Il y a à peu près autant de femmes que d'hommes gestionnaires qui ont participé à notre recherche. Pour préserver I'anonymat des personnes, nous avons utilisé le masculin. 
3. Étant donné que la très grande majorité des intervenants sont des femmes, nous avons mis ce mot au féminin.

4. Les tableaux 4 et 5 ne reproduisent pas la fréquence des activités. Le lecteur qui désire plus d'information devra se référer à la recherche de Béliveau et Poulin (juillet 1991).

\section{Références bibliographiques}

Beaudoin, A., M. Poulin et F. TuRGeOn-KraWCZUK (1991). " Regards sur les nouvelles technologies et leur signification pour l'intervention et la formation en service social ", Intervention, $\mathrm{n}^{\circ}$ 88, mars, 71-82.

BéLIVEAU, G., M. POULIN, en coll. avec G. BEAUdOIN (1991). Informatisation des dossiers de service social en milieu hospitalier : processus d'implantation et impacts. Tome I : Cadre conceptuel et méthodologie de la recherche. Laboratoire de recherche, École de service social, Université Laval, juillet, $96 \mathrm{p}$.

BéliVeAU, G., M. Poulin, en coll. avec G. BeAudoIn (1991). Inforrnatisation des dossiers de service social en milieu hospitalier : processus d'implantation et impacts. Tome II : Processus d'implantation de l'informatique. Laboratoire de recherche, École de service social, Université Laval, juillet, $103 \mathrm{p}$.

BéliVeAu, G., M. Poulin, en coll. avec G. BeAudoIn (1991). Informatisation des dossiers de service social en milieu hospitalier : processus d'implantation et impacts. Tome III : Impacts de l'informatisation. Laboratoire de recherche, École de service social, Université Laval, juillet, $73 \mathrm{p}$.

BéliveAu, G., M. POULIN, en coll. avec G. BeAudoin (1991). Les perceptions et les attitudes des intervenants sociaux du Québec au sujet de l'utilisation de l'informatique dans leur milieu de travail. Recherche subventionnée par le Conseil de la recherche en sciences humaines du Canada (CRSH). Université Laval, École de service social, juillet, $204 \mathrm{p}$.

COTTER, B. (1981). Planning and Implementing Social Service Information System : A Guide for Management and Users, Human Services, Monograph Series, Project Share, $\mathrm{n}^{\circ} 25$, sept., $193 \mathrm{p}$.

DURIBREUX, F. (1988). Informatique et travail social. Paris : Syros/Alternatives, $159 \mathrm{p}$.

GANDY, J.M. et A. DJAO (1987). "Les réactions du personnel à l'introduction et à l'utilisation de l'informatique dans les organismes de service social », Service social, vol. 36, $\mathrm{n}^{\circ} 1$ : 33-53.

GANDY, J.M. et L. TEPPERMAN (1990). False Alarm : the Computerization of Eight Social Welfare Organizations, Wilfrid Laurier University Press, $220 \mathrm{p}$.

Glastonbury, B. et S. TOOLE (1990). A Casebook of Computer Applications in the Social and Human Services, W. Le Mendola, Ed.

HURTUBISE, R. (1984). La bureautique - Éléments et impacts. Montréal : Éditions Agence d'Arc inc., 75 p. 
LARSEN, J.K. (1987). "Implementing Computers in Mental Health Settings ", Computers in Human Services, vol. 2, $\mathrm{n}^{\text {os }}$ 3/4 : 145-170.

MouliN, B. (1986). "Les systèmes de base de connaissance dans les organisations », L'ingénieur, sept.-oct. : 21-27.

Poulin, M., A. BeAudoin, G. Béliveau, A. LAMARre et F. TURGEON-KRAWCZUK (1991). Les effets et l'appréciation de l'utilisation d'un logiciel interactif par les parents d'enfants atteints de fibrose kystique. Laboratoire de recherche, École de service social, Université Laval, novembre, 182 p.

POULIN, M. et G. BÉliveAu (1987). L'utilisation et le développement de l'informatique dans les services sociaux. Synthèse critique $\mathrm{n}^{\circ} 27, \mathrm{Com}$ mission d'enquête sur les services de santé et les services sociaux, Publications du Québec.

SCHOECH, D.J. (1990). Human Services Computing Concepts \& Applications. Series Editor : Simon Slavin, The Haworth Press, $626 \mathrm{p}$.

SCHOECH, D.J., L.L. SCHKADE et R.S. MAYERS (1981). "Strategies for Information System Development ", Administration in Social Work, vol. 5, $n^{\text {os }} 3 / 4: 11-26$.

YIN, Robert K. (1984). Case Study Research : Design and Methods. Beverly Hills, London, New Delhi : Sage Publications, 159 p. 